\title{
THE SOLID-STATE INCLUSION COMPLEX BETWEEN THE MONO-ANION OF CALIX[4]ARENE AND PROTONATED DIAMINO-BICYCLOUNDECANE
}

\author{
Oleksandr Shkurenko ${ }^{\mathrm{a}}$, Kinga Suwinska ${ }^{\mathrm{a}}$, Florent Perret ${ }^{\mathrm{b}}$, Katia Sigaud $^{\mathrm{c}}$, \\ Antoine Leydier ${ }^{c}$, Said Jebors ${ }^{c}$ and Anthony W. Coleman ${ }^{\mathrm{c}^{*}}$ \\ ${ }^{a}$ Institute of Physical Chemistry, Polish Academy of Sciences, Kasprzaka, 44/52, PL-01 224 Warsaw, Poland \\ ${ }^{b}$ Laboratoire de Chimie Organique 2, ICBMS, CNRS UMR 5246, Université Lyon 1, 43, bd du 11 novembre 1918, 69622 \\ Villeurbanne cedex, France \\ 'Institut de Biologie et Chimie des Protéines, UMR 5086, CNRS, Université Lyon 1, 7 passage du Vercors, F69367 Lyon, France. \\ Email: aw.coleman@ibcp.fr
}

\begin{abstract}
Diamino-bicycloundecane mono-deprotonates H-calix[4]arene and the solid-structure of the resulting salt has been determined by X-ray crystallography. Two different complexes are present in the structure with the Diamino-bicycloundecane cation held in the calix[4]arene cavity by CH-p interactions. In the packing there is a hydrogen-bonded polymeric chain. Both the complexes formed layered structures with differing inter-layer distances.
\end{abstract}

Keywords: Calix[n]arenes, X-ray Crystallography, Structure, Mono-anionic, Diamino-Bicycloundecane, inclusion polymer.

\section{Introduction}

The calix[n]arenes are a class of macrocycle organic host compounds widely studied for their complexation properties [1], they have been shown to complex molecules at sizes varying from metallic cations [2], anions [3], small organic molecules [4] through peptides [5] to proteins [6] and DNA [7]. As they possess two different chemistries: at the para-aromatic position and at the phenolic hydroxyl position, their selective chemical modifycation may be achieved [8], and hence groups with divergent chemical and physical properties may be readily introduced at either face.

A wide range of amphiphilic calix[n]arene derivatives have been developed by modification at one or two faces. We have some time been interested in the synthesis of amphiphilic calix[n]arenes having a geometry tuned to the formation of micellar structures, such molecules are based mono-functionalisation at the phenolic face followed by use of quinine-methide substitution at the three remaining phenolic groups [9]. Obviously the key step is the initial mono-substitution.

The synthesis of such molecules has been studied by Casnati et al. [10] via the 1-3 dialkoxy or tetraalkoxycalix[4]arenes followed by hydrolysis with respectively 1 or 3 equivalents of trimethylsilyl iodide. We have previously demonstrated mono-substitution of calix[4]arene using potassium carbonate as a base, however yields are very low, ca $10 \%$. Kalchenko has used sodium hydroxide as a base with DMSO as a solvent for monosubstitution [11]. Work by Groenen et al. [12] showed that cesium fluoride or potassium fluoride could be used as suitable mono-alkylating agents. An interesting article by Cunningham et al. showed that certain organic bases such as diamino-bicycloundecane (DBU) could be used, with total selectivity to mono-deprotonate all the calix[n]arenes [13].

\section{Experimental}

Suitable crystals for X-ray diffraction, were grown from an acetonitrile solution of a 1:1 mixture of calix[4]arene and diamino-bicycloundecane by slow evaporation.

The crystal collection and refinement data for 1 are given in Table 1.

$\mathrm{H}$ atoms were treated as riding atoms in geometrically idealized positions, with $\mathrm{C}-\mathrm{H}$ distances of 0.95 (aromatic ring), $0.99\left(\mathrm{CH}_{2}\right), 0.98\left(\mathrm{CH}_{3}\right), 0.88(\mathrm{NH})$ and $0.84 \AA(\mathrm{OH})$, and with $U_{\text {iso }}(\mathrm{H})=k U_{\text {eq }}(\mathrm{C}, \mathrm{O})$, where $\mathrm{k}=1.5$ for the methyl and hydroxyl groups and $\mathrm{k}=1.2$ otherwise.

Data collection: COLLECT [14]; cell refinement and data reduction: DENZO and SCALEPACK [15]; programs used to solve structure and refine structure: SHELXS97 and SHELXL97 [16]; molecular graphics: Diamond 3.1f [17]. 


\section{Results and Discussion}

While the yields obtained from the use of DBU as a selective mono-deprotonation agent for calix[4]arene are too low to allow its use in the production of mono-O-alkylated calix[4]arene derivatives, during the course of the study suitable crystals for crystallographic study were obtained by slow evaporation from a chloroform-methanol solvent mixture.

Table 1

Crystal data and structure refinement details of 1.DBU

\begin{tabular}{|c|c|}
\hline & 1.DBU \\
\hline Empirical formula & $\mathrm{C}_{28} \mathrm{H}_{23} \mathrm{O}_{4}^{-} \cdot \mathrm{C}_{9} \mathrm{H}_{17} \mathrm{~N}_{2}^{+} \cdot 0.5 \mathrm{CH}_{3} \mathrm{OH}$ \\
\hline Formula weight & 592.73 \\
\hline Temperature (K) & $100(2)$ \\
\hline Diffractometer & Nonius KappaCCD \\
\hline Wavelength $(\AA)$ & 0.71073 \\
\hline Crystal & colourless \\
\hline Crystal size $\left(\mathrm{mm}^{3}\right)$ & $0.45 \times 0.40 \times 0.30$ \\
\hline Crystal system & Monoclinic \\
\hline Space group & $P 2_{1} / c$ \\
\hline \multicolumn{2}{|l|}{ Unit cell dimension } \\
\hline$a(\AA)$ & $16.9981(2)$ \\
\hline$b(\AA)$ & $19.1230(2)$ \\
\hline$c(\AA)$ & $19.7663(2)$ \\
\hline$\alpha\left(^{\circ}\right)$ & 90 \\
\hline$\beta\left(^{\circ}\right)$ & $107.8250(4)$ \\
\hline$\gamma\left({ }^{\circ}\right)$ & 90 \\
\hline Volume $\left(\AA^{3}\right)$ & $6116.7(1)$ \\
\hline$Z$ & 8 \\
\hline Calculated density $\left(\mathrm{g} \cdot \mathrm{cm}^{-3}\right)$ & 1.287 \\
\hline$F(000)$ & 2536 \\
\hline Absorption coefficient $\left(\mathrm{mm}^{-1}\right)$ & 0.084 \\
\hline$\theta$ Range for data collection $\left({ }^{\circ}\right)$ & $2.97-27.46$ \\
\hline$h k l$ Ranges & $-22 \leq h \leq 22,-24 \leq k \leq 24,-25 \leq l \leq 25$ \\
\hline Reflections collected/unique & 90818 / 13966 \\
\hline Completeness (\%) to $\theta$ & $99.9 / 27.46$ \\
\hline Absorption correction & None \\
\hline Refinement method & Full-matrix least-squares on $\mathrm{F}^{2}$ \\
\hline Data/restraints / parameters & $13966 / 0 / 802$ \\
\hline Goodness-of-fit on $F^{2}$ & 1.06 \\
\hline Final $R$ indices $[I>2 \sigma(I)]$ & $R=0.041, w R=0.090$ \\
\hline$R$ indices (all data) & $R=0.049, w R=0.094$ \\
\hline
\end{tabular}




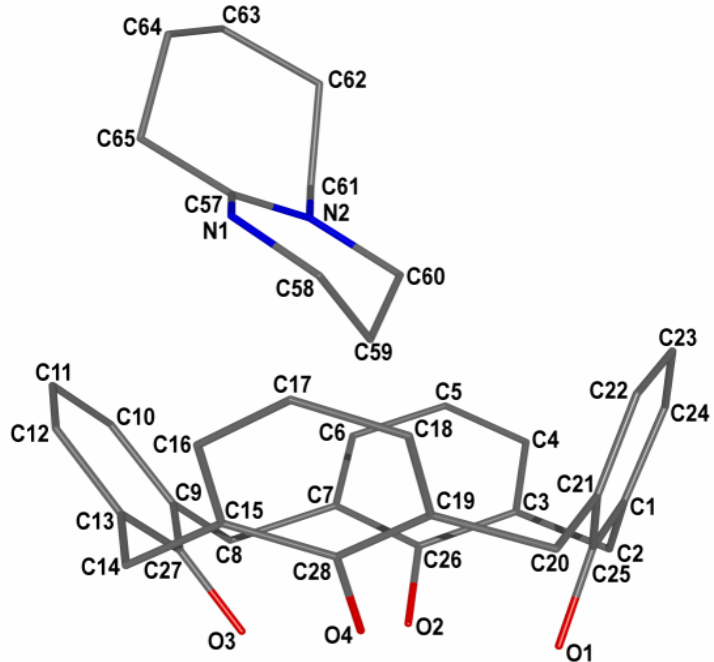

075

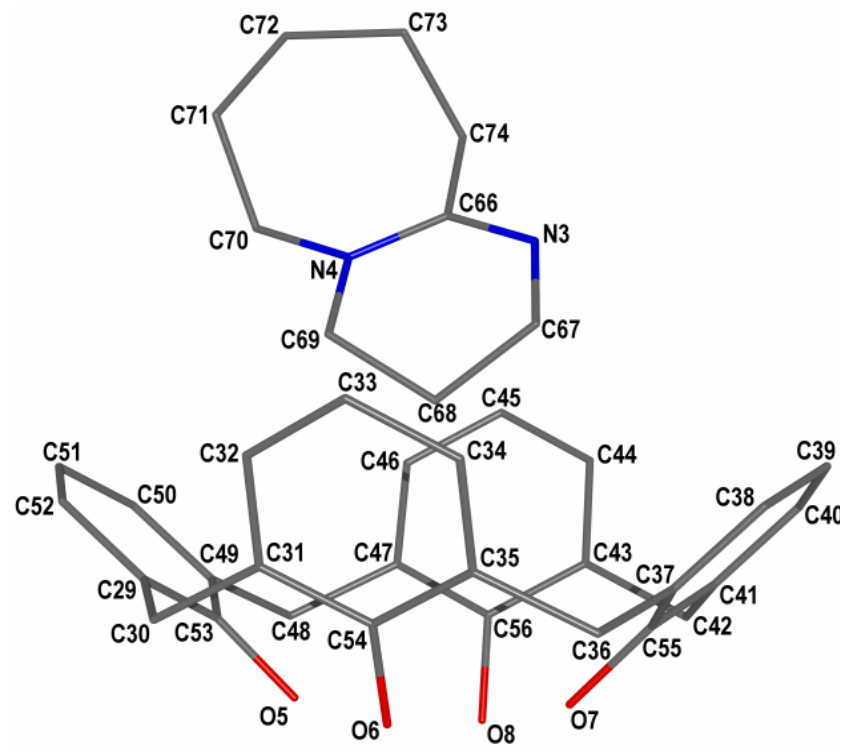

b

Figure 1. The molecular structures of Complexes I and II along with the atom labelling.

The solid-state structure shows a 2:2 complex of two mono-anions of calix[4]arene 1 with two protonated DBU cations and a single molecule of methanol present as a solvate in the structure.

The two different complexes are given, along with atom labelling in Figure $1 \mathbf{a}$ and $\mathbf{b}$.

Bond Lengths are given in Table 1 and Bond Angles in Table 2 (for non-hydrogen atoms), atomic coordinates are given in the Supplementary data.

Table 2

\section{Bond length $(\AA)$}

\begin{tabular}{llll}
\hline Bond & $d, \AA$ & Bond & $d, \AA$ \\
\hline $\mathrm{O} 1-\mathrm{C} 25$ & $1.373(2)$ & $\mathrm{C} 35-\mathrm{C} 54$ & $1.408(2)$ \\
$\mathrm{O} 2-\mathrm{C} 26$ & $1.362(2)$ & $\mathrm{C} 35-\mathrm{C} 36$ & $1.517(2)$ \\
$\mathrm{O} 3-\mathrm{C} 27$ & $1.356(2)$ & $\mathrm{C} 36-\mathrm{C} 37$ & $1.518(2)$ \\
$\mathrm{O} 4-\mathrm{C} 28$ & $1.372(1)$ & $\mathrm{C} 37-\mathrm{C} 38$ & $1.399(2)$ \\
$\mathrm{C} 1-\mathrm{C} 24$ & $1.399(2)$ & $\mathrm{C} 37-\mathrm{C} 55$ & $1.401(2)$ \\
$\mathrm{C} 1-\mathrm{C} 25$ & $1.405(2)$ & $\mathrm{C} 38-\mathrm{C} 39$ & $1.387(2)$ \\
$\mathrm{C} 1-\mathrm{C} 2$ & $1.522(2)$ & $\mathrm{C} 39-\mathrm{C} 40$ & $1.386(2)$ \\
$\mathrm{C} 2-\mathrm{C} 3$ & $1.521(2)$ & $\mathrm{C} 40-\mathrm{C} 41$ & $1.395(2)$ \\
$\mathrm{C} 3-\mathrm{C} 4$ & $1.397(2)$ & $\mathrm{C} 41-\mathrm{C} 55$ & $1.409(2)$ \\
$\mathrm{C} 3-\mathrm{C} 26$ & $1.402(2)$ & $\mathrm{C} 41-\mathrm{C} 42$ & $1.517(2)$ \\
$\mathrm{C} 4-\mathrm{C} 5$ & $1.390(2)$ & $\mathrm{C} 42-\mathrm{C} 43$ & $1.518(2)$ \\
$\mathrm{C} 5-\mathrm{C} 6$ & $1.387(2)$ & $\mathrm{C} 43-\mathrm{C} 44$ & $1.398(2)$ \\
$\mathrm{C} 6-\mathrm{C} 7$ & $1.394(2)$ & $\mathrm{C} 43-\mathrm{C} 56$ & $1.400(2)$ \\
$\mathrm{C} 7-\mathrm{C} 26$ & $1.406(2)$ & $\mathrm{C} 44-\mathrm{C} 45$ & $1.389(2)$ \\
$\mathrm{C} 7-\mathrm{C} 8$ & $1.518(2)$ & $\mathrm{C} 45-\mathrm{C} 46$ & $1.385(2)$
\end{tabular}




\begin{tabular}{|c|c|c|c|}
\hline $\mathrm{C} 8-\mathrm{C} 9$ & $1.521(2)$ & $\mathrm{C} 46-\mathrm{C} 47$ & $1.399(2)$ \\
\hline $\mathrm{C} 9-\mathrm{C} 10$ & $1.395(2)$ & $\mathrm{C} 47-\mathrm{C} 56$ & $1.401(2)$ \\
\hline $\mathrm{C} 9-\mathrm{C} 27$ & $1.409(2)$ & $\mathrm{C} 47-\mathrm{C} 48$ & $1.523(2)$ \\
\hline $\mathrm{C} 10-\mathrm{C} 11$ & $1.390(2)$ & $\mathrm{C} 48-\mathrm{C} 49$ & $1.514(2)$ \\
\hline $\mathrm{C} 11-\mathrm{C} 12$ & $1.387(2)$ & $\mathrm{C} 49-\mathrm{C} 50$ & $1.398(2)$ \\
\hline $\mathrm{C} 12-\mathrm{C} 13$ & $1.395(2)$ & $\mathrm{C} 49-\mathrm{C} 53$ & $1.404(2)$ \\
\hline $\mathrm{C} 13-\mathrm{C} 27$ & $1.410(2)$ & $\mathrm{C} 50-\mathrm{C} 51$ & $1.387(2)$ \\
\hline $\mathrm{C} 13-\mathrm{C} 14$ & $1.518(2)$ & C51-C52 & $1.387(2)$ \\
\hline $\mathrm{C} 14-\mathrm{C} 15$ & $1.519(2)$ & $\mathrm{N} 1-\mathrm{C} 57$ & $1.320(2)$ \\
\hline $\mathrm{C} 15-\mathrm{C} 16$ & $1.397(2)$ & $\mathrm{N} 1-\mathrm{C} 58$ & $1.464(2)$ \\
\hline $\mathrm{C} 15-\mathrm{C} 28$ & $1.404(2)$ & $\mathrm{N} 2-\mathrm{C} 57$ & $1.325(2)$ \\
\hline $\mathrm{C} 16-\mathrm{C} 17$ & $1.389(2)$ & $\mathrm{N} 2-\mathrm{C} 60$ & $1.480(2)$ \\
\hline $\mathrm{C} 17-\mathrm{C} 18$ & $1.387(2)$ & $\mathrm{N} 2-\mathrm{C} 61$ & $1.480(2)$ \\
\hline $\mathrm{C} 18-\mathrm{C} 19$ & $1.391(2)$ & $\mathrm{C} 57-\mathrm{C} 65$ & $1.498(2)$ \\
\hline $\mathrm{C} 19-\mathrm{C} 28$ & $1.400(2)$ & C58-C59 & $1.517(2)$ \\
\hline $\mathrm{C} 19-\mathrm{C} 20$ & $1.519(2)$ & $\mathrm{C} 59-\mathrm{C} 60$ & $1.515(2)$ \\
\hline $\mathrm{C} 20-\mathrm{C} 21$ & $1.522(2)$ & $\mathrm{C} 61-\mathrm{C} 62$ & $1.525(2)$ \\
\hline $\mathrm{C} 21-\mathrm{C} 22$ & $1.393(2)$ & $\mathrm{C} 62-\mathrm{C} 63$ & $1.527(2)$ \\
\hline $\mathrm{C} 21-\mathrm{C} 25$ & $1.405(2)$ & C63-C64 & $1.526(2)$ \\
\hline $\mathrm{C} 22-\mathrm{C} 23$ & $1.389(2)$ & C64-C65 & $1.539(2)$ \\
\hline $\mathrm{C} 23-\mathrm{C} 24$ & $1.391(2)$ & $\mathrm{N} 3-\mathrm{C} 66$ & $1.316(2)$ \\
\hline $\mathrm{O} 5-\mathrm{C} 53$ & $1.355(2)$ & N3-C67 & $1.469(2)$ \\
\hline $\mathrm{O} 6-\mathrm{C} 54$ & $1.350(1)$ & $\mathrm{N} 4-\mathrm{C} 66$ & $1.324(2)$ \\
\hline $\mathrm{O} 7-\mathrm{C} 55$ & $1.371(2)$ & $\mathrm{N} 4-\mathrm{C} 70$ & $1.475(2)$ \\
\hline $\mathrm{O} 8-\mathrm{C} 56$ & $1.381(2)$ & $\mathrm{N} 4-\mathrm{C} 69$ & $1.478(2)$ \\
\hline $\mathrm{C} 29-\mathrm{C} 52$ & $1.397(2)$ & $\mathrm{C} 66-\mathrm{C} 74$ & $1.498(2)$ \\
\hline $\mathrm{C} 29-\mathrm{C} 53$ & $1.406(2)$ & С67-C68 & $1.512(2)$ \\
\hline $\mathrm{C} 29-\mathrm{C} 30$ & $1.515(2)$ & C68-C69 & $1.516(2)$ \\
\hline $\mathrm{C} 30-\mathrm{C} 31$ & $1.517(2)$ & $\mathrm{C} 70-\mathrm{C} 71$ & $1.526(2)$ \\
\hline $\mathrm{C} 31-\mathrm{C} 32$ & $1.393(2)$ & $\mathrm{C} 71-\mathrm{C} 72$ & $1.527(2)$ \\
\hline $\mathrm{C} 31-\mathrm{C} 54$ & $1.410(2)$ & $\mathrm{C} 72-\mathrm{C} 73$ & $1.525(2)$ \\
\hline $\mathrm{C} 32-\mathrm{C} 33$ & $1.387(2)$ & $\mathrm{C} 73-\mathrm{C} 74$ & $1.540(2)$ \\
\hline $\mathrm{C} 33-\mathrm{C} 34$ & $1.391(2)$ & $\mathrm{C} 75-\mathrm{O} 9$ & $1.404(2)$ \\
\hline $\mathrm{C} 34-\mathrm{C} 35$ & $1.392(2)$ & & \\
\hline
\end{tabular}

An expected the cone conformations of the calix[4]arene mono-anions are stabilized by hydrogen bonds at the phenolic face, distances 2.703(1), 2.524(1), and 2.521(1) $\AA$ for anion I and 2.559(1), 2.587(1), and 2.788(1) $\AA$ for anion II. In both cases the two shorter hydrogen bond lengths are associated with the deprotonated phenolic groups. 
Angles between bonds ( $\AA$ )

\begin{tabular}{|c|c|c|c|}
\hline Angle & $\angle,^{\circ}$ & Angle & $\angle,^{\circ}$ \\
\hline $\mathrm{C} 24-\mathrm{C} 1-\mathrm{C} 25$ & $118.1(1)$ & $\mathrm{C} 38-\mathrm{C} 37-\mathrm{C} 36$ & $119.5(1)$ \\
\hline $\mathrm{C} 24-\mathrm{C} 1-\mathrm{C} 2$ & $120.0(1)$ & $\mathrm{C} 55-\mathrm{C} 37-\mathrm{C} 36$ & $122.0(1)$ \\
\hline $\mathrm{C} 25-\mathrm{C} 1-\mathrm{C} 2$ & $121.8(1)$ & $\mathrm{C} 39-\mathrm{C} 38-\mathrm{C} 37$ & $121.4(1)$ \\
\hline $\mathrm{C} 3-\mathrm{C} 2-\mathrm{C} 1$ & 111.1(1) & $\mathrm{C} 40-\mathrm{C} 39-\mathrm{C} 38$ & 119.3(1) \\
\hline $\mathrm{C} 4-\mathrm{C} 3-\mathrm{C} 26$ & $118.5(1)$ & $\mathrm{C} 39-\mathrm{C} 40-\mathrm{C} 41$ & $121.4(1)$ \\
\hline $\mathrm{C} 4-\mathrm{C} 3-\mathrm{C} 2$ & $120.3(1)$ & $\mathrm{C} 40-\mathrm{C} 41-\mathrm{C} 55$ & $118.5(1)$ \\
\hline $\mathrm{C} 26-\mathrm{C} 3-\mathrm{C} 2$ & $121.2(1)$ & $\mathrm{C} 40-\mathrm{C} 41-\mathrm{C} 42$ & $120.6(1)$ \\
\hline $\mathrm{C} 5-\mathrm{C} 4-\mathrm{C} 3$ & $121.0(1)$ & $\mathrm{C} 55-\mathrm{C} 41-\mathrm{C} 42$ & $120.8(1)$ \\
\hline $\mathrm{C} 6-\mathrm{C} 5-\mathrm{C} 4$ & $119.6(1)$ & $\mathrm{C} 41-\mathrm{C} 42-\mathrm{C} 43$ & $111.8(1)$ \\
\hline $\mathrm{C} 5-\mathrm{C} 6-\mathrm{C} 7$ & $121.3(1)$ & $\mathrm{C} 44-\mathrm{C} 43-\mathrm{C} 56$ & $118.5(1)$ \\
\hline $\mathrm{C} 6-\mathrm{C} 7-\mathrm{C} 26$ & $118.3(1)$ & $\mathrm{C} 44-\mathrm{C} 43-\mathrm{C} 42$ & $120.0(1)$ \\
\hline $\mathrm{C} 6-\mathrm{C} 7-\mathrm{C} 8$ & $120.3(1)$ & $\mathrm{C} 56-\mathrm{C} 43-\mathrm{C} 42$ & $121.5(1)$ \\
\hline $\mathrm{C} 26-\mathrm{C} 7-\mathrm{C} 8$ & $121.3(1)$ & $\mathrm{C} 45-\mathrm{C} 44-\mathrm{C} 43$ & $120.8(1)$ \\
\hline $\mathrm{C} 7-\mathrm{C} 8-\mathrm{C} 9$ & $115.6(1)$ & $\mathrm{C} 46-\mathrm{C} 45-\mathrm{C} 44$ & $119.5(1)$ \\
\hline $\mathrm{C} 10-\mathrm{C} 9-\mathrm{C} 27$ & 119.1(1) & $\mathrm{C} 45-\mathrm{C} 46-\mathrm{C} 47$ & $121.6(1)$ \\
\hline $\mathrm{C} 10-\mathrm{C} 9-\mathrm{C} 8$ & 119.9(1) & $\mathrm{C} 46-\mathrm{C} 47-\mathrm{C} 56$ & $117.9(1)$ \\
\hline $\mathrm{C} 27-\mathrm{C} 9-\mathrm{C} 8$ & $121.0(1)$ & $\mathrm{C} 46-\mathrm{C} 47-\mathrm{C} 48$ & $120.0(1)$ \\
\hline $\mathrm{C} 11-\mathrm{C} 10-\mathrm{C} 9$ & $121.1(1)$ & $\mathrm{C} 56-\mathrm{C} 47-\mathrm{C} 48$ & $122.1(1)$ \\
\hline $\mathrm{C} 12-\mathrm{C} 11-\mathrm{C} 10$ & $119.5(1)$ & $\mathrm{C} 49-\mathrm{C} 48-\mathrm{C} 47$ & $112.5(1)$ \\
\hline $\mathrm{C} 11-\mathrm{C} 12-\mathrm{C} 13$ & $121.2(1)$ & $\mathrm{C} 50-\mathrm{C} 49-\mathrm{C} 53$ & $118.5(1)$ \\
\hline $\mathrm{C} 12-\mathrm{C} 13-\mathrm{C} 27$ & 119.1(1) & $\mathrm{C} 50-\mathrm{C} 49-\mathrm{C} 48$ & $122.0(1)$ \\
\hline $\mathrm{C} 12-\mathrm{C} 13-\mathrm{C} 14$ & $119.9(1)$ & $\mathrm{C} 53-\mathrm{C} 49-\mathrm{C} 48$ & $119.6(1)$ \\
\hline $\mathrm{C} 27-\mathrm{C} 13-\mathrm{C} 14$ & $121.1(1)$ & $\mathrm{C} 51-\mathrm{C} 50-\mathrm{C} 49$ & $121.0(1)$ \\
\hline $\mathrm{C} 13-\mathrm{C} 14-\mathrm{C} 15$ & $114.1(1)$ & $\mathrm{C} 50-\mathrm{C} 51-\mathrm{C} 52$ & 119.7(1) \\
\hline $\mathrm{C} 16-\mathrm{C} 15-\mathrm{C} 28$ & $118.6(1)$ & $\mathrm{C} 51-\mathrm{C} 52-\mathrm{C} 29$ & $121.3(1)$ \\
\hline $\mathrm{C} 16-\mathrm{C} 15-\mathrm{C} 14$ & $120.3(1)$ & $\mathrm{O} 5-\mathrm{C} 53-\mathrm{C} 49$ & $116.5(1)$ \\
\hline $\mathrm{C} 28-\mathrm{C} 15-\mathrm{C} 14$ & 121.1(1) & $\mathrm{O} 5-\mathrm{C} 53-\mathrm{C} 29$ & $122.2(1)$ \\
\hline $\mathrm{C} 17-\mathrm{C} 16-\mathrm{C} 15$ & $120.9(1)$ & $\mathrm{C} 49-\mathrm{C} 53-\mathrm{C} 29$ & $121.3(1)$ \\
\hline $\mathrm{C} 18-\mathrm{C} 17-\mathrm{C} 16$ & $119.4(1)$ & $\mathrm{O} 6-\mathrm{C} 54-\mathrm{C} 35$ & $120.2(1)$ \\
\hline $\mathrm{C} 17-\mathrm{C} 18-\mathrm{C} 19$ & $121.6(1)$ & $\mathrm{O} 6-\mathrm{C} 54-\mathrm{C} 31$ & $120.7(1)$ \\
\hline $\mathrm{C} 18-\mathrm{C} 19-\mathrm{C} 28$ & $118.4(1)$ & $\mathrm{C} 35-\mathrm{C} 54-\mathrm{C} 31$ & $119.2(1)$ \\
\hline $\mathrm{C} 18-\mathrm{C} 19-\mathrm{C} 20$ & $120.2(1)$ & $\mathrm{O} 7-\mathrm{C} 55-\mathrm{C} 37$ & $122.0(1)$ \\
\hline $\mathrm{C} 28-\mathrm{C} 19-\mathrm{C} 20$ & $121.4(1)$ & $\mathrm{O} 7-\mathrm{C} 55-\mathrm{C} 41$ & $117.2(1)$ \\
\hline $\mathrm{C} 19-\mathrm{C} 20-\mathrm{C} 21$ & $112.6(1)$ & $\mathrm{C} 37-\mathrm{C} 55-\mathrm{C} 41$ & $120.9(1)$ \\
\hline $\mathrm{C} 22-\mathrm{C} 21-\mathrm{C} 25$ & $118.7(1)$ & $\mathrm{O} 8-\mathrm{C} 56-\mathrm{C} 43$ & 119.7(1) \\
\hline $\mathrm{C} 22-\mathrm{C} 21-\mathrm{C} 20$ & $120.4(1)$ & $\mathrm{O} 8-\mathrm{C} 56-\mathrm{C} 47$ & $118.8(1)$ \\
\hline $\mathrm{C} 25-\mathrm{C} 21-\mathrm{C} 20$ & $120.9(1)$ & $\mathrm{C} 43-\mathrm{C} 56-\mathrm{C} 47$ & $121.6(1)$ \\
\hline $\mathrm{C} 23-\mathrm{C} 22-\mathrm{C} 21$ & $121.2(1)$ & $\mathrm{C} 57-\mathrm{N} 1-\mathrm{C} 58$ & $123.4(1)$ \\
\hline $\mathrm{C} 22-\mathrm{C} 23-\mathrm{C} 24$ & $119.4(1)$ & $\mathrm{C} 57-\mathrm{N} 2-\mathrm{C} 60$ & $120.6(1)$ \\
\hline $\mathrm{C} 23-\mathrm{C} 24-\mathrm{C} 1$ & $121.4(1)$ & $\mathrm{C} 57-\mathrm{N} 2-\mathrm{C} 61$ & $121.6(1)$ \\
\hline $\mathrm{O} 1-\mathrm{C} 25-\mathrm{C} 21$ & $120.3(1)$ & $\mathrm{C} 60-\mathrm{N} 2-\mathrm{C} 61$ & $117.7(1)$ \\
\hline $\mathrm{O} 1-\mathrm{C} 25-\mathrm{C} 1$ & $118.5(1)$ & $\mathrm{N} 1-\mathrm{C} 57-\mathrm{N} 2$ & $122.1(1)$ \\
\hline $\mathrm{C} 21-\mathrm{C} 25-\mathrm{C} 1$ & $121.2(1)$ & $\mathrm{N} 1-\mathrm{C} 57-\mathrm{C} 65$ & $117.5(1)$ \\
\hline $\mathrm{O} 2-\mathrm{C} 26-\mathrm{C} 3$ & $117.9(1)$ & $\mathrm{N} 2-\mathrm{C} 57-\mathrm{C} 65$ & $120.3(1)$ \\
\hline $\mathrm{O} 2-\mathrm{C} 26-\mathrm{C} 7$ & $120.9(1)$ & $\mathrm{N} 1-\mathrm{C} 58-\mathrm{C} 59$ & $108.7(1)$ \\
\hline $\mathrm{C} 3-\mathrm{C} 26-\mathrm{C} 7$ & 121.1(1) & $\mathrm{C} 60-\mathrm{C} 59-\mathrm{C} 58$ & $109.5(1)$ \\
\hline $\mathrm{O} 3-\mathrm{C} 27-\mathrm{C} 9$ & $120.3(1)$ & $\mathrm{N} 2-\mathrm{C} 60-\mathrm{C} 59$ & $110.2(1)$ \\
\hline $\mathrm{O} 3-\mathrm{C} 27-\mathrm{C} 13$ & $119.6(1)$ & $\mathrm{N} 2-\mathrm{C} 61-\mathrm{C} 62$ & $114.2(1)$ \\
\hline $\mathrm{C} 9-\mathrm{C} 27-\mathrm{C} 13$ & $120.1(1)$ & $\mathrm{C} 61-\mathrm{C} 62-\mathrm{C} 63$ & $115.2(1)$ \\
\hline $\mathrm{O} 4-\mathrm{C} 28-\mathrm{C} 19$ & $117.5(1)$ & $\mathrm{C} 64-\mathrm{C} 63-\mathrm{C} 62$ & $115.3(1)$ \\
\hline $\mathrm{O} 4-\mathrm{C} 28-\mathrm{C} 15$ & $121.4(1)$ & C63-C64-C65 & $113.5(1)$ \\
\hline
\end{tabular}




\begin{tabular}{|c|c|c|c|}
\hline $\mathrm{C} 19-\mathrm{C} 28-\mathrm{C} 15$ & 121.1(1) & $\mathrm{C} 57-\mathrm{C} 65-\mathrm{C} 64$ & $112.9(1)$ \\
\hline $\mathrm{C} 52-\mathrm{C} 29-\mathrm{C} 53$ & $118.2(1)$ & $\mathrm{C} 66-\mathrm{N} 3-\mathrm{C} 67$ & $124.3(1)$ \\
\hline $\mathrm{C} 52-\mathrm{C} 29-\mathrm{C} 30$ & $120.9(1)$ & $\mathrm{C} 66-\mathrm{N} 4-\mathrm{C} 70$ & $121.8(1)$ \\
\hline $\mathrm{C} 53-\mathrm{C} 29-\mathrm{C} 30$ & $120.9(1)$ & $\mathrm{C} 66-\mathrm{N} 4-\mathrm{C} 69$ & $120.3(1)$ \\
\hline $\mathrm{C} 29-\mathrm{C} 30-\mathrm{C} 31$ & $114.3(1)$ & $\mathrm{C} 70-\mathrm{N} 4-\mathrm{C} 69$ & $117.9(1)$ \\
\hline $\mathrm{C} 32-\mathrm{C} 31-\mathrm{C} 54$ & $119.3(1)$ & $\mathrm{N} 3-\mathrm{C} 66-\mathrm{N} 4$ & $122.1(1)$ \\
\hline $\mathrm{C} 32-\mathrm{C} 31-\mathrm{C} 30$ & $119.7(1)$ & $\mathrm{N} 3-\mathrm{C} 66-\mathrm{C} 74$ & $117.5(1)$ \\
\hline $\mathrm{C} 54-\mathrm{C} 31-\mathrm{C} 30$ & 121.1(1) & $\mathrm{N} 4-\mathrm{C} 66-\mathrm{C} 74$ & $120.4(1)$ \\
\hline $\mathrm{C} 33-\mathrm{C} 32-\mathrm{C} 31$ & $121.8(1)$ & N3-C67-C68 & $109.5(1)$ \\
\hline $\mathrm{C} 32-\mathrm{C} 33-\mathrm{C} 34$ & $118.8(1)$ & $\mathrm{C} 67-\mathrm{C} 68-\mathrm{C} 69$ & $110.2(1)$ \\
\hline $\mathrm{C} 33-\mathrm{C} 34-\mathrm{C} 35$ & 121.1(1) & N4-C69-C68 & $110.3(1)$ \\
\hline $\mathrm{C} 34-\mathrm{C} 35-\mathrm{C} 54$ & $119.9(1)$ & $\mathrm{N} 4-\mathrm{C} 70-\mathrm{C} 71$ & $113.4(1)$ \\
\hline $\mathrm{C} 34-\mathrm{C} 35-\mathrm{C} 36$ & $120.0(1)$ & $\mathrm{C} 70-\mathrm{C} 71-\mathrm{C} 72$ & $113.7(1)$ \\
\hline $\mathrm{C} 54-\mathrm{C} 35-\mathrm{C} 36$ & $120.0(1)$ & $\mathrm{C} 73-\mathrm{C} 72-\mathrm{C} 71$ & $114.6(1)$ \\
\hline $\mathrm{C} 35-\mathrm{C} 36-\mathrm{C} 37$ & $113.0(1)$ & $\mathrm{C} 72-\mathrm{C} 73-\mathrm{C} 74$ & $114.0(1)$ \\
\hline $\mathrm{C} 38-\mathrm{C} 37-\mathrm{C} 55$ & $118.5(1)$ & $\mathrm{C} 66-\mathrm{C} 74-\mathrm{C} 73$ & $112.2(1)$ \\
\hline
\end{tabular}

The DBU cation I is included within the calix[4]arene $\mathbf{I}$ cavity. There are $\mathrm{CH}^{\cdots} \cdots \pi$ interactions between the two hydrogen atoms of the most deeply inserted methylene group, distances of 3.691(2) and 3.572(2) $\AA$ and two further interactions between one hydrogen of the other two methylene groups and the other two aromatic rings, distance of 3.604(2) and 3.666(2) $\AA$. The protonated nitrogen of this DBU cation is hydrogen bonded to the deprotonated phenolic hydroxyl group of anion II, $\mathrm{N}-\mathrm{H} \cdots \mathrm{O}$ distance 2.791(1) $\AA$.

In the case of the second DBU cation (II) $\mathrm{CH}^{\cdots} \pi \pi$ interactions are stronger (3.459(2), 3.345(2), 3.572(2), 3.546(2) $\AA$ ) which correlates with more flattened conformation for II (cone angles of 55.34(3), 86.34(3) ${ }^{\circ}$ for I and $43.27,86.67^{\circ}$ for $\left.\mathbf{I I}\right)$. The methanol molecule forms hydrogen bond with deprotonated hydroxyl group of I, so DBU cation connects with other hydroxyl group via hydrogen bond of 2.916(1) $\AA$.

As a result of $\mathrm{N}-\mathrm{H} \cdots \mathrm{O}$ bonding and $\mathrm{CH} \cdots \pi$ interactions (Table 3 ) each DBU cation binds two neighbouring calixarenes (Fig. 2). DBU cations present in the crystal structure as racemic mixture, so both complexes $\mathbf{I}$ and $\mathbf{I I}$ are

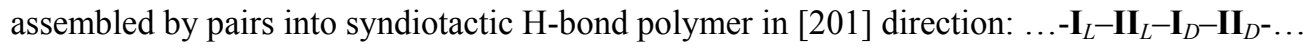

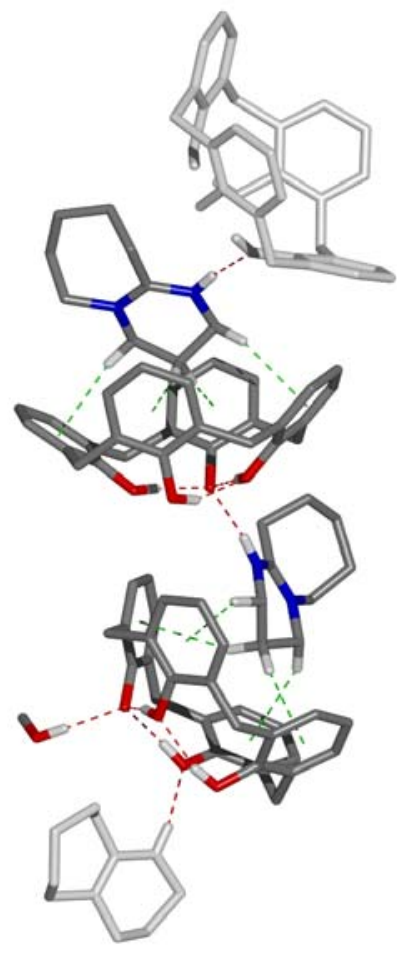

Figure 2. Hydrogen-bonded polymeric chain of 1. Protons not involved in intermolecular interactions or hydrogen bonding have been removed for clarity. 
The distance between methanol molecules corresponds to the polymer link length (17.00 $\AA$ ). All polymeric chains which lie in $x z$ plane are parallel, so folded layers consisted of complexes I and II are anti-parallel (Figure 3a). Hydrogen bonds and contacts, as listed in Table 3, link the units in the chain, and then the inter-chains cohesion is via held electrostatic and van der Waals interactions. The layer formed by calixarenes $\mathbf{I}$ is thinner than that formed by calixarenes II due to the presence of a methanol molecule (Figure 3b).

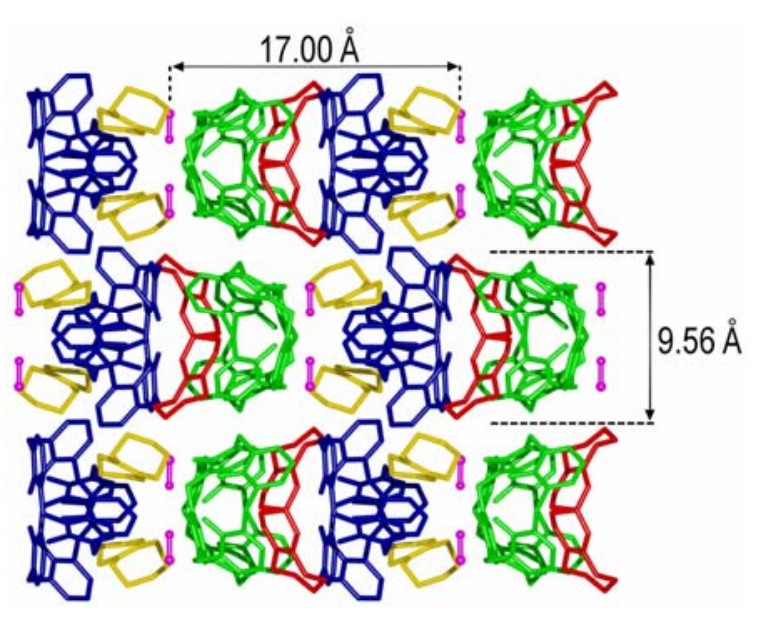

$\mathbf{a}$

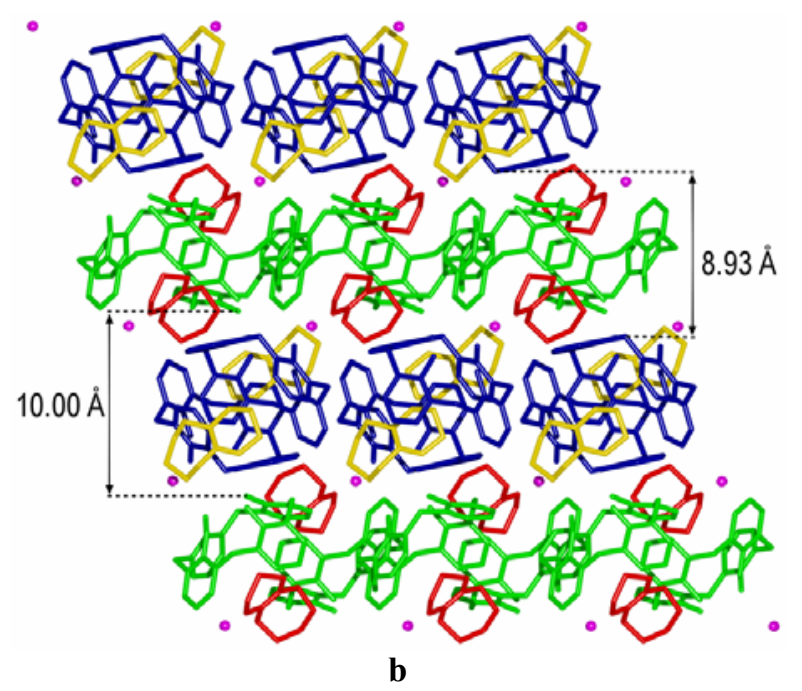

b

Figure 3. The packing diagrams of the complexes 1. Hydrogen atoms have been removed for clarity.

Hydrogen-bond and $\mathrm{C}-\mathrm{H} \cdots \pi$ contact geometry

Table 3

\begin{tabular}{|c|c|c|c|c|}
\hline $\mathrm{D}-\mathrm{H} \cdots \mathrm{A}$ & $\mathrm{D}-\mathrm{H}, \AA$ & $\mathrm{H} \cdots \mathrm{A}, \AA$ & $\mathrm{D} \cdots \mathrm{A}, \AA$ & $\mathrm{D}-\mathrm{H} \cdots \mathrm{A},{ }^{\circ}$ \\
\hline $\mathrm{O} 1-\mathrm{H} 1 \cdots \mathrm{O} 4$ & 0.84 & 1.89 & $2.703(1)$ & 163 \\
\hline $\mathrm{O} 2-\mathrm{H} 2 \cdots \mathrm{O} 3$ & 0.84 & 1.70 & $2.524(1)$ & 168 \\
\hline $\mathrm{O} 4-\mathrm{H} 4 \cdots \mathrm{O} 3$ & 0.84 & 1.70 & $2.521(1)$ & 167 \\
\hline $\mathrm{O} 5-\mathrm{H} 5 \mathrm{~A} \cdots \mathrm{O} 6$ & 0.84 & 1.74 & $2.559(1)$ & 163 \\
\hline $\mathrm{O} 7-\mathrm{H} 7 \cdots \mathrm{O} 6$ & 0.84 & 1.76 & $2.587(1)$ & 167 \\
\hline $\mathrm{O} 8-\mathrm{H} 8 \cdots \mathrm{O} 7$ & 0.84 & 1.99 & $2.787(1)$ & 158 \\
\hline $\mathrm{N} 1-\mathrm{H} 1 \cdots \mathrm{O}^{\mathrm{i}}$ & 0.88 & 1.92 & $2.791(1)$ & 173 \\
\hline $\mathrm{N} 3-\mathrm{H} 3 \cdots \mathrm{O} 2^{\mathrm{ii}}$ & 0.88 & 2.07 & $2.916(1)$ & 162 \\
\hline $\mathrm{O} 9-\mathrm{H} 9 \cdots \mathrm{O} 3$ & 0.84 & 1.90 & $2.741(1)$ & 179 \\
\hline $\mathrm{C} 2-\mathrm{H} 2 \mathrm{~A} \cdots \mathrm{O} 9^{\mathrm{iii}}$ & 0.99 & 2.66 & $3.631(1)$ & 166 \\
\hline $\mathrm{C} 16-\mathrm{H} 16 \cdots \mathrm{O} 5^{\mathrm{iv}}$ & 0.95 & 2.65 & $3.542(1)$ & 157 \\
\hline $\mathrm{C} 74-\mathrm{H} 74 \mathrm{~B} \cdots \mathrm{O} 3^{\mathrm{ii}}$ & 0.99 & 2.60 & $3.533(1)$ & 158 \\
\hline C58-H58A $\cdots \pi 4$ & 0.99 & 2.72 & $3.691(1)$ & 168 \\
\hline С $59-\mathrm{H} 59 \mathrm{~A} \cdots \pi 1$ & 0.99 & 2.74 & $3.707(1)$ & 165 \\
\hline С $59-$ Н59В $\cdots \pi 3$ & 0.99 & 2.73 & $3.722(1)$ & 176 \\
\hline $\mathrm{C} 60-\mathrm{H} 60 \mathrm{~B} \cdots \pi 2$ & 0.99 & 2.80 & $3.774(1)$ & 167 \\
\hline C65-H65A $\cdots \pi 6^{\text {iv }}$ & 0.99 & 2.66 & $3.594(1)$ & 157 \\
\hline С67-H67B $\cdots \pi 7$ & 0.99 & 2.78 & $3.765(1)$ & 173 \\
\hline C68-H68A $\cdots \pi 6$ & 0.99 & 2.40 & $3.380(1)$ & 172 \\
\hline С68-H68B $\cdots \pi 8$ & 0.99 & 2.49 & $3.438(1)$ & 160 \\
\hline С69-H69A $\cdots \pi 5$ & 0.99 & 2.74 & $3.646(1)$ & 152 \\
\hline
\end{tabular}

Symmetry codes: (i) $-\mathrm{x}+1,-\mathrm{y}+1,-\mathrm{z}+1$; (ii) $-\mathrm{x}, \mathrm{y}+1 / 2,-\mathrm{z}+1 / 2$; (iii) $\mathrm{x}, 1 / 2-\mathrm{y},-1 / 2+\mathrm{z}$; (iv) 1-x, 1-y, 1-z.

Phenolic ring name in table above is defined by oxygen atom label, for example $\pi 1$ means phenolic ring with O1 atom. 


\section{References}

[1]. Gutsche, C.D. Calixarenes Revisited; Royal Society of Chemistry: Cambridge, 1998.

[2]. a) Tu, C.; Surowiec, K.; Bartsch, R.A. Tetrahedron. 2007, 63, 4184-4189; b) Yaftian, M.R.; Razipour, M.R.; Matt, D. JRNC 2006, 270 (2), 357-361; c) Marcos, P. M.; Felix, S.; Ascenso, J.R.; Segurado, M.A.P.; Mellah, B.; Abidi, R.; Hubscher-Bruder, V.; Arnaud-Neu, F. Supramol. Chem. 2006, 18 (2), 147-159.

[3]. a) Klimentova, J.; Vojtisek, P. J. Mol. Struct. 2007, 826(1), 48-63; b) Delaigue, X.; Gutsche, C. D.; Harrowfield, J.M.; Ogden, M.I.; Skelton, B.W.; Stewart, D.F.; White, A.H. Supramol. Chem. 2004, 16(8), 603-609.

[4]. Mutihac, L.; Buschmann, H.-J.; Tudorescu, A.; Mutihac, R. J. Incl. Phenom. Macroc. Chem. 2003 , 47 (3-4), $123-128$.

[5]. Douteau-Guevel, N.; Perret, F.; Coleman, A.W.; Morel, J.-P.; Morel-Desrosiers, N. J. Chem. Soc., Perkin Trans. 2 2002, 524-532.

[6]. a) Coleman, A.W. ; Perret, F. ; Cecillon, S. ; Moussa, A. ; Martin, A. ; Dupin, M. ; Perron, H. New J. Chem. 2007, 31(5), 711-717; b) Perret, F. ; Lazar, A.N. ; Coleman, A.W. Chem. Commun. 2006, (23), 2425-2438; c) da Silva, E.; Lazar, A.N.; Coleman, A.W. J. Drug Delivery Sci. and Technol. 2004, 14(1), 3-20; d) Memmi, L.; Lazar, A.N.; Brioude, A.; Ball, V.; Coleman, A.W. Chem. Commun. 2001, (23), 2474-2475; e) Oh, S.W.; Moon, J.D.; Lim, H.J.; Park, S.Y.; Kim, T.; Park, J.B.; Han, M.H.; Synyder, M.; Choi, E.Y. FASEB J. 2005, 19(10), 1335-1337.

[7]. a) Zadmard, R.; Schrader, T. Angew. Chem., Int.Ed. 2006, 45(17), 2703-2706; b) Dudic, M.; Colombo, A.; Sansone, F.; Casnati, A.; Donofrio, G.; Ungaro, R. Tetrahedron 2004, 60(50), 11613-11618.

[8]. Thorndorf, I.; Shivanyuk, A.; Böhmer, V. Chapter 2 in Calixarenes 2001, eds Asfari, Z.; Böhmer, V.; Harrowfield, J.; Vicens, J.; Kluwer Academic Publishers: Dordrecht, 2001; pp 26-53.

[9]. a) Suwinska, K.; Shkurenko, O.; Mbemba, C.; Leydier, A.; Jebors, S.; Coleman, A.W.; Matar, R.; Falson, P. New J. Chem. 2008, 32, 1988-1998; b) Mbemba, C.; Sigaud, K.; Perret, F.; Suwinska, K.; Shkurenko, O.; Coleman, A.W. J. Incl. Phenom. Macroc. Chem 2008, 61(1-2), 29-40.

[10]. A.Casnati, A. Pochini, Andrea; R. Ungaro, R. Cacciapaglia, L. Mandolini, J. Chem. Soc., Perkin Trans. 1 1991, (8), 2052-2054.

[11]. Boyko, V.I.; Podoprigorina, A.A.; Yakovenko, A.V.; Pirozhenko, V.V.; Kalchenko, V.I. J. Incl. Phenom. and Macroc. Chem. 2004, 50(3-4), 193-197.

[12]. Groenen, L.C.; Ruel, B.H.M.; Casnati, A.; Verboom, W.; Pochini, A.; Ungaro, R.; Reinhoudt, D.N. Tetrahedron 1991, 47(39), 8379-8384.

[13]. Cunningham, I.D.; Woolfall, M. J. Org. Chem. 2005, 70(23), 9248-9256.

[14]. Nonius. COLLECT. Nonius BV, Delft 1998, The Netherlands.

[15]. Otwinowski, Z.; Minor, W. Methods in Enzymology. Vol. 276, Macromolecular Crystallography, Part A, ed. Carter, C.W. Jr; Sweet, R. M. New York: Academic Press 1997; pp. 307-326.

[16]. Sheldrick, G. M. Acta Cryst. 2008, A64, 112-122.

[17]. Pennington, W.T. J. Appl. Cryst. 1999, 32, 1028-1029. 